\title{
THE FIRST TWO OBSTRUCTIONS TO THE FREENESS OF ARRANGEMENTS
}

\author{
SERGEY YUZVINSKY
}

\begin{abstract}
In his previous paper the author characterized free arrangements by the vanishing of cohomology modules of a certain sheaf of graded modules over a polynomial ring. Thus the homogeneous components of these cohomology modules can be viewed as obstructions to the freeness of an arrangement. In this paper the first two obstructions are studied in detail. In particular the component of degree zero of the first nontrivial cohomology module has a close relation to formal arrangements and to the operation of truncation. This enables us to prove that in dimension greater than two every free arrangement is formal and not a proper truncation of an essential arrangement.
\end{abstract}

\section{INTRODUCTION}

In this paper we develop further the ideas from [Y] where certain sheaf cohomology was applied to the theory of arrangements.

Let $V$ be an $l$-dimensional $(l \geq 2)$ vector space over an arbitrary field $K$ and $\mathscr{A}$ an arrangement of $n \quad(n \geq 2)$ hyperplanes in $V$. For each $H \in \mathscr{A}$ we fix a linear functional $\alpha_{H} \in V^{*}$ such that $\operatorname{ker} \alpha_{H}=H$. Let $S=\bigoplus_{d \geq 0} S_{d}$ be the graded symmetric algebra of $V^{*}$ and $\operatorname{Der} S=\operatorname{Der}_{K}(S, S)$ the graded $S$-module of derivations of $S$. The following graded submodule of Der $S$ was introduced by $\mathrm{H}$. Terao [T1, T3] and studied in many papers on arrangements: $D=D(\mathscr{A})=\left\{\theta \in \operatorname{Der}(S) \mid \theta\left(\alpha_{H}\right) \in S \alpha_{H}, H \in \mathscr{A}\right\}$. If the module $D$ is free then the arrangement $\mathscr{A}$ is called free.

Let $L_{0}$ be the lattice of all intersections of hyperplanes from $\mathscr{A}$ except the smallest one $\bigcap_{H \in \mathscr{A}} H$, ordered by inclusion. Every $X \in L_{0}$ defines the subarrangement $\mathscr{A}_{X}=\{H \in \mathscr{A} \mid H \supset X\}$ of $\mathscr{A}$ and hence an $S$-module $D(X)=D\left(\mathscr{A}_{X}\right)$. These modules with the natural inclusions form a sheaf $\mathscr{D}$ on $L_{0}$. It was proved in [Y] that if $\mathscr{A}$ is essential (i.e., $\bigcap_{H \in \mathscr{A}} H=0$ ) and free then $H^{i}\left(L_{0}, \mathscr{D}\right)=0$ for every $i, 0<i<l-1$. In fact the results of [Y] yield a necessary and sufficient condition for the freeness of $\mathscr{A}$ in terms of the vanishing of certain cohomology of $\mathscr{D}$ (see $\S 1$ ).

The main goal of this paper is to make use of the graded structure of the modules $H^{i}\left(L_{0}, \mathscr{D}\right)=\bigoplus_{d \geq 0} H_{d}^{i}$. As it was also proved in [Y] if $\mathscr{A}$ is essential then these modules have finite length. Thus the finite set of spaces $H_{d}^{i}, 1 \leq$ $i \leq l-2$, can be viewed as obstructions to the freeness of $\mathscr{A}$. Here we study the first two obstructions $H_{0}^{1}$ and $H_{1}^{1}$. The former of these obstructions turns

Received by the editors October 4, 1990.

1980 Mathematics Subject Classification (1985 Revision). Primary 05B35, 32C40, 13 C15. 
out to be very closely related to the property of $\mathscr{A}$ to be formal (which was introduced and studied in [FR]) and to the operation of truncation (considered in [Z]). More precisely if $\mathscr{A}$ is essential then $\operatorname{dim} H_{0}^{1}+l$ is the maximum dimension of an essential arrangement which has a truncation isomorphic to $\mathscr{A}$ (see $\S \S 2$ and 3). In particular this enables us to prove the implication "free $\Rightarrow$ formal" (which solves a problem "of significant interest" from [FR]) and a conjecture of Ziegler's that free arrangements are not proper truncations. We also give an example of two combinatorially equivalent arrangements one of which is formal and the other is not. This answers negatively the question from [FR] whether being formal is a combinatorial property. ${ }^{1}$

The latter obstruction $H_{1}^{1}$ is studied in $\S 4$. We consider there the 1-dimensional simplicial complex $C$ formed by the elements of $L_{0}$ of codimension 1 and 2. We also consider all linear dependences of length 3 among $\alpha_{H}$. The $n$ tuples of coefficients of these dependences may in turn be dependent. We denote by $T$ the linear space formed by the coefficient sequences of those "secondary" dependences. Then we construct a linear map $\nu_{*}: T \otimes V \rightarrow H_{1}(C)$ and prove that $H_{1}^{1}$ vanishes if and only if $\nu_{*}$ is surjective. This result gives a new example of an interaction among different facets of arrangements and may be useful as an alternative approach to free arrangements.

\section{Notation}

Here we recall some definitions and set up the notation for the rest of the paper. Throughout, $\mathscr{A}$ will be an $l$-arrangement of $n$ hyperplanes in a linear space $V$ (of dimension $l$ ) over an arbitrary field $K$ and for every $H \in \mathscr{A}_{H}$, $\alpha_{H}$ will be a fixed linear functional such that $\operatorname{ker} \alpha_{H}=H$. $\mathscr{A}$ is called Boolean if all the functionals $\alpha_{H}$ are linearly independent. If $\mathscr{A}_{1}$ and $\mathscr{A}_{2}$ are arrangements in spaces $V_{1}$ and $V_{2}$ respectively then their direct sum is the arrangement in $V_{1} \oplus V_{2}$ given by

$$
\mathscr{A}_{1} \oplus \mathscr{A}_{2}=\left\{H \oplus V_{2} \mid H \in \mathscr{A}_{1}\right\} \cup\left\{V_{1} \oplus H^{\prime} \mid H^{\prime} \in \mathscr{A}_{2}\right\} .
$$

If an arrangement is not linearly isomorphic to any nontrivial direct sum it is called irreducible.

The graded ring $S$ and the graded module $D=D(\mathscr{A})$ were defined in the introduction. There exist several equivalent definitions of $D$. We will use that $D$ is naturally isomorphic to the $S$-module of all linear maps $V^{*} \rightarrow S$ which send $\alpha_{H}$ into $S \alpha_{H}$ for every $H \in \mathscr{A}$.

The intersection lattice of $\mathscr{A}$ ordered by reverse inclusion is denoted by $L$. The minimal element of $L$ is $V$, its maximal element $\bigcap_{H \in \mathscr{A}} H$ is denoted by $U$. If $U=0$ the arrangement $\mathscr{A}$ is called essential. The lattice $L$ is ranked and the rank of an element $X \in L$ coincides with its codimension in $V$ which we denote by $\operatorname{codim} X$. In particular the rank of $L$ is the codimension of $U$ which we denote by $m$. We have $m=l$ if and only if $\mathscr{A}$ is essential. We will also use the lattice $L^{o p}$ which coincides with $L$ as the set and is provided with the reverse order (i.e., the inclusion) and the poset $L_{0}=L^{o p} \backslash\{U\}$. Not to confuse the orders we use only the symbol $\subset$ for the order on $L^{o p}$.

\footnotetext{
${ }^{1}$ As it was pointed out to me by the referee the priority here belongs to Ziegler whose example [Z, Example 8.7] can be used to the same effect. This raises an interesting question about relations between the formality and the degree sequences from $[\mathrm{Z}]$.
} 
Every $X \in L$ defines the subarrangement $\mathscr{A}_{X}$ of $\mathscr{A}$ which consists of the hyperplanes containing $X$. We put $D(X)=D\left(\mathscr{A}_{X}\right)$. The set $\left\{\alpha_{H} \mid H \in \mathscr{A}_{X}\right\}$ generates Ann $X=\left\{\alpha \in V^{*} \mid \alpha(v)=0\right.$ for every $\left.v \in X\right\}$. The part of $L_{0}$ for $\mathscr{A}_{X}$ is played by the poset $L_{X}=\left\{Y \in L_{0} \mid Y \supset X, Y \neq X\right\}$. Clearly $L_{U}=L_{0}$. Since every poset $L_{X}$ has the unique maximal element $V$ its chain complex is a simplicial cone. In particular $L_{X}$ is acyclic, i.e., its cohomology groups with any (constant) coefficients vanish in positive dimensions.

If $Q$ is an arbitrary finite poset then we view it as a topological space with the topology consisting of all increasing subsets of $Q$, i.e,. subsets $R$ such that if $X \in R$ and $Y \geq X$ then $Y \in R$. The category of sheaves (of $S$-modules or linear spaces) on this topological space is isomorphic to the category of covariant functors on $Q$. In particular a sheaf $\mathscr{F}$ on $Q$ is defined by the stalks $\mathscr{F}(X)$, $X \in Q$, and by the structure morphisms $\rho_{Y, X}: \mathscr{F}(X) \rightarrow \mathscr{F}(Y), X, Y \in Q$, $X<Y$, such that $\rho_{Y, Z} \rho_{X, Y}=\rho_{X, Z}$ for every $X<Y<Z$. A global section $s$ of $\mathscr{F}$ is a collection $(s(X) \in \mathscr{F}(X))_{X \in Q}$ such that $\rho_{X, Y}(s(X))=s(Y)$ for every $X<Y$. The set ( $S$-module or linear space) of all global sections of $\mathscr{F}$ is denoted by $\Gamma(\mathscr{F})$. The set of sections on a subset $R$ of $Q$ is denoted by $\Gamma(R, \mathscr{F})$. A sheaf $\mathscr{F}$ on $Q$ induces a sheaf on any subset of $Q$ and we will keep the symbol $\mathscr{F}$ for this sheaf. All information we need about the cohomology of sheaves can be found in [G]. In particular we will use that for every sheaf $\mathscr{F}$ on $Q$ there is a natural isomorphism $H^{0}(Q, \mathscr{F})=\Gamma(\mathscr{F})$.

An example of a sheaf important for arrangement theory is given by the sheaf $\mathscr{D}$ on $L_{0}$ defined by the stalks $\mathscr{D}(X)=D(X)$ and by the natural embeddings $D(X) \subset D(Y)$ for all $X \subset Y$ from $L_{0}$. It is crucial for this paper that all stalks of $\mathscr{D}$ are graded $S$-modules and the structure homomorphisms have degree 0 . Therefore every cohomology module $H^{i}\left(L_{X}, \mathscr{D}\right)$ is graded too. We will denote the homogeneous component of degree $d$ of this module by $H_{d}^{i}\left(L_{X}, \mathscr{D}\right)$.

\section{A COHOMOLOGY CHARACTERIZATION OF FREE ARRANGEMENTS}

In this section we give a cohomology characterization of free arrangements based on results of $[\mathrm{Y}]$. We also introduce a sheaf $\mathscr{D}^{\prime}$ which will play a leading part in the following sections.

Theorem 1.1. An arrangement $\mathscr{A}$ is free if and only if $H^{i}\left(L_{X}, \mathscr{D}\right)=0$ for every $X \in L$ and every $i, 0<i<\operatorname{codim} X-1$.

Proof. To apply results from [Y] we have to make a transition to essential arrangements. For every $X \subset Y, X, Y \in L$, we denote by $\mathscr{A}_{Y}^{X}$ the arrangement $\left\{H / X \mid H \in \mathscr{A}_{Y}\right\}$ in $V / X$ and notice that the arrangement $\mathscr{A}_{X}^{\prime}=\mathscr{A}_{X}^{X}$ is essential. The following equality is straightforward (cf. [ST, Proposition 5.8]):

$$
D\left(\mathscr{A}_{Y}\right)=\left(D\left(\mathscr{A}_{Y}^{X}\right) \otimes S_{X}\right) \oplus S^{d}
$$

where $S_{X}$ is the symmetric algebra of $X^{*}, d=\operatorname{dim} X$, and $\otimes$ is the tensor multiplication over $K$. Here $D\left(\mathscr{A}_{Y}^{X}\right)$ is viewed as an $S_{V / X}$-module where $S_{V / X}$ is the symmetric algebra of $(V / X)^{*}$ and the first summand in the righthand side of (1.1) gets an $S$-module structure via the natural isomorphism $S=S_{V / X} \otimes S_{X}$. The first important consequence of (1.1) for us is that $\mathscr{A}_{X}$ is free if and only if $\mathscr{A}_{X}^{\prime}$ is free. Denote by $\mathscr{D}^{X}$ the sheaf on $L_{X}$ with the stalk $D\left(\mathscr{A}_{Y}^{X}\right)$ at every $Y \supset X$ and with the natural embeddings. Notice that since $L_{X}$ is acyclic, for the constant sheaf $\widetilde{S}^{d}$ on $L_{X}$ with the stalks $S^{d}$ we have 
$H^{i}\left(L_{X}, \widetilde{S}^{d}\right)=0$ for every $i>0$. Then the second consequence of $(1.1)$ is that $H^{i}\left(L_{X}, \mathscr{D}\right)=H^{i}\left(L_{X}, \mathscr{D}^{X}\right) \otimes S_{X}$ for every $i>0$.

Now we can apply results from [Y]. If $\mathscr{A}$ is free then $\mathscr{A}_{X}$ is free for every $X \in L$ (see [T2]) whence $\mathscr{A}_{X}^{\prime}$ is free. Then [Y, Corollary 2.3] implies that $H^{i}\left(L_{X}, \mathscr{D}^{X}\right)=0$ for every $i$ such that $0<i<\operatorname{codim} X-1$ and thus the same is true for $H^{i}\left(L_{X}, \mathscr{D}\right)$.

On the other hand if $\mathscr{A}$ is not free then we can find $X \in L$ of the smallest codimension such that $\mathscr{A}_{X}$ (and thus $\mathscr{A}_{X}^{\prime}$ ) is not free. According to [Y, Corollary 2.6], $H^{i}\left(L_{X}, \mathscr{D}^{X}\right) \neq 0$ for some positive $i<\operatorname{codim} X-1$. Thus the same is true for $H^{i}\left(L_{X}, \mathscr{D}\right)$ which concludes the proof.

Now let $D(\varnothing)$ be the $S$-module of derivations corresponding to the empty arrangement in $V$. Clearly it is a free module of rank $l$. Let $\widetilde{D(\varnothing)}$ be the constant sheaf on $L_{0}$ with the stalks $D(\varnothing)$ (and identity structure homomorphisms). Notice that $\mathscr{D}$ is a subsheaf of $\widetilde{D(\varnothing)}$ and put $\mathscr{D}^{\prime}=\widetilde{D(\varnothing)} / \mathscr{D}$.

Proposition 1.2. For every $X \in L_{0}$ we have a natural isomorphism $H^{i}\left(L_{X}, \mathscr{D}^{\prime}\right)$ $=H^{i+1}\left(L_{X}, \mathscr{D}\right)$ for $i \geq 1$ and an exact sequence

$$
0 \rightarrow H^{0}\left(L_{X}, \mathscr{D}\right) \rightarrow D(\varnothing) \stackrel{\pi}{\longrightarrow} H^{0}\left(L_{X}, \mathscr{D}^{\prime}\right) \rightarrow H^{1}\left(L_{X}, \mathscr{D}\right) \rightarrow 0
$$

where $\pi$ is the set of natural projections $\pi_{Y}: D(\varnothing) \rightarrow D(\varnothing) / D(Y), Y \in L_{X}$.

Proof. The result follows immediately from the exact cohomology sequence corresponding to the short exact sequence

$$
0 \rightarrow \mathscr{D} \rightarrow \widetilde{D(\varnothing)} \rightarrow \mathscr{D}^{\prime} \rightarrow 0
$$

of sheaves and from the acyclicity of $L_{X}$.

Corollary 1.3. An arrangement $\mathscr{A}$ is free if and only if (i) $H^{i}\left(L_{X}, \mathscr{D}^{\prime}\right)=0$ for every $i \geq 1$ and $X \in L_{0}$ with $\operatorname{codim} X \geq i+3$, and (ii) $\pi: D(\varnothing) \rightarrow H^{0}\left(L_{X}, \mathscr{D}^{\prime}\right)$ is surjective for every $X \in L_{0}$ with $\operatorname{codim} X \geq 3$.

Remark 1.4. The above corollary simplifies significantly for 3-arrangements. A 3-arrangement $\mathscr{A}$ is free if and only if $\pi: D(\varnothing) \rightarrow H^{0}\left(L_{0}, \mathscr{D}^{\prime}\right)$ is surjective.

In the rest of the paper we are concerned only with the condition (ii) of Corollary 1.3.

\section{Global Sections of $\mathscr{D}^{\prime}$ OF DEgRee 0}

In this section we focus our attention on the 0-degree component $\Gamma_{0}\left(\mathscr{D}^{\prime}\right)=$ $H_{0}^{0}\left(L_{0}, \mathscr{D}^{\prime}\right)$ of the $S$-module $\Gamma\left(\mathscr{D}^{\prime}\right)=H^{0}\left(L_{0}, \mathscr{D}^{\prime}\right)$ of global sections. Elements of $\Gamma_{0}\left(\mathscr{D}^{\prime}\right)$ are global sections of the sheaf $\mathscr{C}$ of linear spaces which is the 0 -degree component of the sheaf $\mathscr{D}^{\prime}$. The stalks of $\mathscr{C}$ are given by $\mathscr{C}(X)=V / X$ for every $X \in L_{0}$ and the structure homomorphisms are the natural projections. Also notice that the 0-degree component of $D(\varnothing)$ is $V$. Thus according to Corollary 1.3 if $\mathscr{A}$ is free then the natural map $V \rightarrow \Gamma(\mathscr{C})$ is surjective. Since the kernel of this map is $U$ the surjectivity is equivalent to the isomorphism $V / U \simeq \Gamma(\mathscr{C})$. 
Now we give an explicit description of the space $\Gamma(\mathscr{C})$ and include it in a series of linear spaces defined by $\mathscr{A}$. For that we consider the linear space $E_{0}$ (over $K$ ) with basis $\mathscr{A}$ enumerated in some way. Using this basis we regard elements of $E_{0}$ as $n$-tuples $\left(t_{1}, \ldots, t_{n}\right), t_{i} \in K, i=1, \ldots, n$. Then with each linear dependence among the functionals $\alpha_{H}$ one can associate a linear equation on $t_{i}$. For each $k, 2 \leq k \leq m+1$, we denote by $W_{k}(\mathscr{A})=W_{k}$ the solution space of the linear system of equations corresponding to the dependences among $\alpha_{H}$ of length not greater than $k$. Clearly $W_{2}=E_{0}$. Evaluating $\alpha_{H}$ at vectors of $V$ one obtains an isomorphism $V / U \simeq W_{m+1}$. Similarly for every $X \in L_{0}$ the space $V / X$ is naturally isomorphic to the solution space of the system of linear equations corresponding to the linear dependences among $\alpha_{H}$ for $H \in \mathscr{A}_{X}$. Combining this isomorphisms we obtain an isomorphism $\Gamma(\mathscr{C}) \simeq W_{m}$. Identifying the spaces according to these isomorphisms we have the following inclusions

$$
V / U=W_{m+1} \subset \Gamma(\mathscr{C})=W_{m} \subset W_{m-1} \subset \cdots \subset W_{3} \subset W_{2}=E_{0}
$$

Definition 2.1. An arrangement $\mathscr{A}$ is $k$-generated $(2 \leq k \leq m)$ if $V / U=W_{k}$. Following [FR] we will also call 3-generated arrangements formal.

It is clear from the definition that a $k$-generated arrangement is also $k_{1}$ generated for every $k_{1}>k$. Every arrangement is $(m+1)$-generated and an arrangement is 2-generated if and only if it is Boolean. Also an arrangement is $m$-generated if and only if the natural map $V / U \rightarrow \Gamma(\mathscr{C})$ is an isomorphism. In dimensions greater than 3 the conditions from Definition 2.1 are not equivalent for different $k, 3 \leq k \leq m$. For example, the 4-arrangement given by the functionals $x, y, z, u, x+y+z, y+z+u$ is not formal $\left(\operatorname{dim} W_{3}=6\right)$ but $\operatorname{dim} W_{4}=4$ and the arrangement is 4-generated.

It is interesting to observe that the conditions from Definition 2.1 are not combinatorial. In particular the following example gives the negative answer to the problem from [FR] whether the property of being formal is combinatorial (see also the footnote to the Introduction).

Example 2.2. Suppose that $\operatorname{char}(K)=0$ or is sufficiently large. Define two 3arrangements $\mathscr{A}_{1}$ and $\mathscr{A}_{2}$ by the seven common functionals $\alpha_{1}=x, \alpha_{2}=y$, $\alpha_{3}=z, \alpha_{4}=x+y+z, \alpha_{5}=2 x+y+z, \alpha_{6}=2 x+3 y+z, \alpha_{7}=2 x+3 y+4 z$ and by the two more $\alpha_{8}=3 x+5 z, \alpha_{9}=3 x+4 y+5 z$ for $\mathscr{A}_{1}$ and $\alpha_{8}=x+3 z$, $\alpha_{9}=x+2 y+3 z$ for $\mathscr{A}_{2}$. Then the linear dependences of length 3 among the functionals produce the following systems of linear equations with respect to nine unknowns $t_{1}, \ldots, t_{9}$ :

$$
\left.\begin{array}{r}
t_{1}+t_{4}-t_{5}=0 \\
2 t_{2}+t_{5}-t_{6}=0 \\
3 t_{3}+t_{6}-t_{7}=0 \\
3 t_{1}+5 t_{3}-t_{8}=0 \\
4 t_{2}+t_{8}-t_{9}=0 \\
t_{4}+t_{7}-t_{9}=0
\end{array}\right\}
$$


for $\mathscr{A}_{1}$ and

$$
\left.\begin{array}{r}
t_{1}+t_{4}-t_{5}=0 \\
2 t_{2}+t_{5}-t_{6}=0 \\
3 t_{3}+t_{6}-t_{7}=0 \\
t_{1}+3 t_{3}-t_{8}=0 \\
2 t_{2}+t_{8}-t_{9}=0 \\
t_{4}-t_{7}+t_{9}=0
\end{array}\right\}
$$

for $\mathscr{A}_{2}$. One can easily see that the dimension of the solution space for the system (2.2) is 3 while for the system $(2.3)$ it is 4 . Thus $\mathscr{A}_{1}$ is formal while $\mathscr{A}_{2}$ is not. On the other hand the one-to-one correspondence between $\mathscr{A}_{1}$ and $\mathscr{A}_{2}$ given by the enumeration of the functionals generates an isomorphism between their intersection lattices.

For our next result we need a stronger version of the property of being formal.

Definition 2.3. An arrangement $\mathscr{A}$ is called locally formal if $\mathscr{A}_{X}$ is formal for every $X \in L$ with $\operatorname{codim} X \geq 3$.

Notice that locally formal arrangements with $m \geq 3$ are formal and these properties coincide if $m=3$. For higher ranks, arrangements can be formal but not locally formal. An example of the kind is given by linear forms $x, y$, $z, x+y+z, u, x+u, x+y+u, x+y+z+u$. This arrangement is formal but its subarrangement $x, y, z, x+y+z$ defined by the $u$-axis is not.

Proposition 2.4. Assume that $m \geq 3$. Then $\mathscr{A}$ is locally formal if and only if

$$
V / X \simeq \Gamma\left(L_{X}, \mathscr{C}\right)
$$

for every $X \in L$ with $\operatorname{codim} X \geq 3$.

Proof. Suppose $\mathscr{A}$ is locally formal and $X \in L$ with $m_{X}=\operatorname{codim} X \geq 3$. Then $\mathscr{A}_{X}$ is formal, i.e., 3-generated and hence $m_{X}$-generated which is equivalent to (2.4).

Now assume that (2.4) holds for every $X \in L$ with $\operatorname{codim} X \geq 3$. We apply induction on $m(=\operatorname{codim} U)$. If $m=3$ then the statement is trivially true. Suppose $m>3$. By the inductive hypothesis every $\mathscr{A}_{X}$ is formal for $X \in L_{0}$. This implies that $W_{3}=W_{m}$. Since (2.4) implies that $W_{m}=V / U$ we have $W_{3}=V / U$. This means that $\mathscr{A}$ is formal and completes the proof.

The following corollary solves a problem from [FR].

Corollary 2.5. If $m \geq 3$ and $\mathscr{A}$ is free then it is locally formal and hence formal. Proof. If $\mathscr{A}$ is free then $\mathscr{A}_{X}$ is free for every $X \in L$ (see [T2]). Now the result follows immediately from Corollary 1.3 and Proposition 2.4.

\section{TRuncations}

This section explores the relation between the operation of truncation and the space $\Gamma(\mathscr{C})$.

Definition 3.1. Let $\mathscr{A}$ be an $l$-arrangement in a space $V$ and $Z$ a linear subspace of $V$ of dimension $k \geq 2$ in general position with respect to $\mathscr{A}$ in 
the sense that $\operatorname{codim}_{Z}(Z \cap X)=\operatorname{codim} X$ for every $X \in L$ with $\operatorname{codim} X \leq k$. Then the arrangement $\left.\mathscr{A}\right|_{Z}=\{H \cap Z \mid H \in \mathscr{A}\}$ in $Z$ is called the truncation of $\mathscr{A}$ by $Z$ or just a $k$-truncation of $\mathscr{A}$. If $k<l$ we call a $k$-truncation proper.

One can easily give the above definition in terms of functionals $\alpha_{H}$ where $H \in \mathscr{A}$. First of all a subspace $Z$ is in general position with respect to $\mathscr{A}$ if and only if the restriction of the functionals $\alpha_{H}$ to $Z$ does not create new linear dependences among them of length less than $k+1$. Then $\left.\mathscr{A}\right|_{Z}$ can be given by the restrictions of the functionals $\alpha_{H}$ to $Z$. We will always mean these restrictions talking about the functionals corresponding to $\left.\mathscr{A}\right|_{Z}$. Using notation from $\S 2$ we have $W_{p}\left(\left.\mathscr{A}\right|_{z}\right)=W_{p}(\mathscr{A})$ for every $p \leq k$.

The intersection lattice of $\left.\mathscr{A}\right|_{Z}$ is the subposet of $L$ of all elements of rank less then $k$ completed with the unique maximal element. Thus on the level of lattices, Definition 3.1 coincides with the definition of truncation in $[Z, p$. 119]. These definitions also coincide for 3-arrangements and for $k=l-1$. In general our definition of general position with respect to an arrangement is more demanding than that in $[Z]$.

Definition 3.2. Let $\mathscr{A}$ be an $l$-arrangement with $l \geq 2$. Put $W=W_{m}=\Gamma(\mathscr{C})$ for this arrangement. For each $H \in \mathscr{A}$ we define the linear functional $\bar{\alpha}_{H}$ on $W$ by

$$
\bar{\alpha}_{H}(s)=\alpha_{H}(s(H)), \quad \text { for every } s \in W .
$$

Recall that $s(H) \in V / H$ and the right-hand side of (3.1) is well defined since $\alpha_{H}$ can be considered as a map $V / H \rightarrow K$. The arrangement defined in $W$ by all the functionals $\bar{\alpha}_{H} \quad(H \in \mathscr{A})$ will be denoted by $\mathscr{A}$.

\section{Theorem 3.3.}

(i) The arrangement $\overline{\mathscr{A}}$ is essential.

(ii) If $\mathscr{A}$ is essential then $\left.\overline{\mathscr{A}}\right|_{V}=\mathscr{A}$ and $\overline{\mathscr{A}}$ is l-generated.

(iii) If $\mathscr{A}$ is a truncation of an essential arrangement $\mathscr{B}$ in a space $Z$ then there is a linear embedding $Z \subset W$ such that the restriction of $\overline{\mathscr{A}}$ to the image of $Z$ is linearly isomorphic to $\mathscr{B}$.

Proof. (i) Suppose that $s \in W$ and $\bar{\alpha}_{H}(s)=0$ for every $H \in \mathscr{A}$. Then (3.1) implies that $s(H)=0$ for every $H \in \mathscr{A}$. Therefore for every $X \in L_{0}$ we have $s(X) \in \bigcap_{H \in \mathscr{A} X} H / X=0$. This means that $s=0$ and $\overline{\mathscr{A}}$ is essential.

(ii) Since $\mathscr{A}$ is essential, $U=0$ and $V \subset W$. It follows immediately from (3.1) that the restriction of $\bar{\alpha}_{H}$ to $V$ coincides with $\alpha_{H}$ for every $H \in \mathscr{A}$. Now we need to prove that $V$ is in general position with respect to $\mathscr{A}$. For that fix $H_{1}, \ldots, H_{k}$ from $\mathscr{A}$ such that $k \leq l$ and $\sum_{i=1}^{k} c_{i} \alpha_{i}=0$ where $\alpha_{i}=\alpha_{H_{i}}$ for $i=1, \ldots, k$ and some $c_{i} \in K \backslash\{0\}$. Put $X=\bigcap_{i=1}^{k} H_{i}$ and notice that $\operatorname{codim} X<k \leq l$, i.e., $X \in L_{0}$. Now fix an arbitrary $s \in W$ and $a \in V$ such that $s(X)=a+X$. Since $s$ is a section of $\mathscr{C}$ on $L_{0}$ we have $s\left(H_{i}\right)=a+H_{i}$ for every $i=1, \ldots, k$. Thus

$$
\sum_{i=1}^{k} c_{i} \bar{\alpha}_{i}(s)=\sum_{i=1}^{k} c_{i} \alpha_{i}\left(s\left(H_{i}\right)\right)=\sum_{i=1}^{k} c_{i} \alpha_{i}(a)=0
$$

which proves that $\mathscr{A}=\left.\overline{\mathscr{A}}\right|_{V}$. Then we also have $W_{l}(\overline{\mathscr{A}})=W_{l}(\mathscr{A})=W$ whence $\overline{\mathscr{A}}$ is $l$-generated. 
(iii) Suppose $\mathscr{B}$ is an essential $p$-arrangement in a space $Z, V$ is a subspace of $Z$ in general position with respect to $\mathscr{B}$, and $\mathscr{A}=\left.\mathscr{B}\right|_{V}$. Denote by $L^{\prime}$ the intersection lattice of $\mathscr{B}$. Then for every $X \in L$ there exists $X^{\prime} \in L^{\prime}$ such that $\operatorname{codim}_{Z} X^{\prime}=\operatorname{codim} X$ and $X=X^{\prime} \cap V$. The natural linear map $V / X \rightarrow Z / X^{\prime}$ sending $a+X$ to $a+X^{\prime} \quad(a \in V)$ is clearly an isomorphism and we will identify $V / X$ and $Z / X^{\prime}$ via this map. Now we define a linear map $\phi: Z \rightarrow W$ by $\phi(z)(X)=z+X^{\prime}$ for every $z \in Z$ and $X \in L_{0}$. The check that $\phi(z) \in W$ is straightforward. If $\phi(z)=0$, i.e., $z \in \bigcap_{\operatorname{dim} X^{\prime} \geq p-l, X^{\prime} \in L^{\prime}} X^{\prime}=\bigcap_{H^{\prime} \in \mathscr{B}} H^{\prime}$ then since $\mathscr{B}$ is essential $z=0$. Thus $\phi$ is an embedding of $Z$ in $W$. To prove the last statement fix $H \in \mathscr{B}$, denote by $\beta$ the respect functional and put $\alpha=\left.\beta\right|_{V}$. Then for every $z \in Z$ we have

$$
\bar{\alpha}(\phi(z))=\alpha(\phi(z))(H \cap V)=\alpha(z+(H \cap V))=\beta(z)
$$

and the theorem is proved.

Corollary 3.4. If $\mathscr{A}$ is essential then the dimension of $\Gamma(\mathscr{C})$ is the maximal dimension of essential arrangements which have truncations isomorphic to $\mathscr{A}$.

Corollary 3.5. No m-generated arrangement is a proper truncation of an essential arrangement. In particular due to Corollary 2.5 no free arrangement with $m \geq 3$ is a proper truncation of an essential arrangement.

The second part of Corollary 3.5 has been conjectured by G. Ziegler.

Remark 3.6. Using notation from Theorem 3.3(iii), the image of $Z$ in $W$ may not be in general position to $\overline{\mathscr{A}}$ and $\mathscr{B}$ may not be isomorphic to any truncation of $\mathscr{\mathscr { A }}$. For example, if $\mathscr{B}$ is the 4-arrangement from the example following Definition 2.1 and $\mathscr{A}$ is an arbitrary 3-truncation of $\mathscr{B}$ then $\overline{\mathscr{A}}$ has dimension 6. However $\mathscr{B}$ is 4-generated and hence is not a proper truncation of an essential arrangement.

The above example is possible only because the arrangement $\mathscr{B}$ in it is not 3-generated. Otherwise we have the following generalization of Corollary 3.5.

Proposition 3.7. Let $\mathscr{A}$ be an essential k-generated l-arrangement in a space $V(3 \leq k \leq l)$ and $\mathscr{B}$ a p-truncation of $\mathscr{A}$ with $k \leq p \leq l$. Then $\mathscr{\mathscr { B }}$ is linearly isomorphic to $\mathscr{A}$.

Proof. By definition of $p$-truncation we have $W_{p}(\mathscr{B})=W_{p}(\mathscr{A})$. Since $\mathscr{A}$ is $k$-generated we have $W_{p}(\mathscr{A}) \subset W_{k}(\mathscr{A})=V$ whence $W_{p}(\mathscr{A})=V$. Thus the dimension of $\overline{\mathscr{B}}$ is equal to $l$. Now the result follows immediately from Theorem 3.3(iii).

Corollary 3.8. In dimension greater than 2 a free essential arrangement is defined by any of its 3-truncation up to linear isomorphism.

\section{Global SECtions of $\mathscr{D}^{\prime}$ OF DegReE 1}

In this section we study the map $\pi: D(\varnothing) \rightarrow \Gamma\left(\mathscr{D}^{\prime}\right)$ in positive degrees, mainly in degree 1 . To avoid trivialities we only consider the case where $\mathscr{A}$ is essential and $l \geq 3$. Moreover since our goal is to study obstructions to the freeness of $\mathscr{A}$ we can focus our attention on the even narrower class of arrangements defined by the properties (4.1)-(4.3) below.

First of all, using induction on $l$ we can assume the following (cf. Corollary 1.3). 
(4.1) For every $X \in L_{0}$ with $\operatorname{codim} X \geq 3$ the natural map $\pi_{X}: D(\varnothing) \rightarrow$ $\Gamma\left(L_{X}, \mathscr{D}^{\prime}\right)$ is surjective, i.e., it induces an isomorphism

$$
D(\varnothing) / D(X) \simeq \Gamma\left(L_{X}, \mathscr{D}^{\prime}\right) .
$$

This condition is vacuous if $l=3$. For higher dimensions it allows one to extend sections from the set $L(2)=\left\{X \in L_{0} \mid \operatorname{codim} X \leq 2\right\}$ to $L_{0}$. More precisely (4.1) implies the following.

(4.1)' The restriction $\Gamma\left(\mathscr{D}^{\prime}\right) \rightarrow \Gamma\left(L(2), \mathscr{D}^{\prime}\right)$ is an isomorphism.

Then since we have already studied $\pi$ in degree 0 we can assume that it is surjective in degree 0 . Together with (4.1) it amounts to the following (cf. Proposition 2.4).

(4.2) $\mathscr{A}$ is formal.

Finally we recall that the direct sum of two arrangements is free if and only if the summands are free (cf. [ST, Proposition 5.8]). This allows us to further restrict the class of arrangements.

(4.3) $\mathscr{A}$ is irreducible.

In fact we will not use (4.3) but will need only the following corollary of (4.2) and (4.3). Let $M$ be the set of all $X \in L$ such that $\operatorname{codim} X=2$ and $\left|\mathscr{A}_{X}\right|>2$ (i.e., $\mathscr{A}_{X}$ is dependent). Then (4.2) and (4.3) imply

$$
\text { (4.3) } \bigcup_{X \in M} \mathscr{A}_{X}=\mathscr{A} \text {. }
$$

In the rest of the section we will always assume that the conditions $(4.1)-(4.3)$ hold for $\mathscr{A}$.

Now we introduce the setup for an interpretation of $\Gamma\left(\mathscr{D}^{\prime}\right)$. The union $M \cup \mathscr{A}$ is a subposet of $L$ and we denote by $C$ its (one-dimensional) chain complex over $K$. Thus $C: 0 \rightarrow C_{1} \stackrel{d}{\longrightarrow} C_{0} \rightarrow 0$ where $C_{0}$ is a linear space with the basis $M \cup \mathscr{A}, C_{1}$ is a linear space with the basis $\{(X, H) \mid X \in M$, $\left.H \in \mathscr{A}_{X}\right\}$, and $d(X, H)=H-X$. Notice that in the introduction the same symbol $C$ was used for the simplicial complex on $M \cap \mathscr{A}$ but this ambiguity disappears after passing to homology (see Theorem 4.9).

Now for each $X \in M$ denote by $E_{X}$ the linear space with the basis $\mathscr{A}_{X}$, by $E_{0}$ the linear space with the basis $\mathscr{A}$, and by $\lambda_{X}$ the linear map $E_{X} \rightarrow$ $\operatorname{Ann}(X)$ generated by $H \mapsto \alpha_{H} \quad\left(H \in \mathscr{A}_{X}\right)$. We put also $R_{X}=\operatorname{ker} \lambda_{X}$ and $E_{1}=\bigoplus_{X \in M} R_{X}$. Notice that $C_{1}=\bigoplus_{X \in M} E_{X}$ and thus $E_{1}$ is a subspace of $C_{1}$. The assignment $(X, H) \mapsto H$ defines a linear map $\rho: C_{1} \rightarrow E_{0}$ which is surjective due to (4.3) ${ }^{\prime}$. Putting $\tau=\left.\rho\right|_{E_{1}}$ we get another one-dimensional complex $E: 0 \rightarrow E_{1} \stackrel{\tau}{\longrightarrow} E_{0} \rightarrow 0$. We also put $T=H_{1}(E)=\operatorname{ker} \tau$ and observe that $H_{0}(E)=V^{*}$ because $\mathscr{A}$ is formal and essential and $\rho$ is surjective.

Now we can give a description of $\Gamma=\Gamma\left(\mathscr{D}^{\prime}\right)$ and $\operatorname{Im} \pi$ which will be used in the proof of the main result.

Lemma 4.4. For every $X \in L$ consider the $S$-module $F_{X}$ of all linear maps Ann $X \rightarrow S$ and put $F_{X}^{0}=\left\{s \in F_{X} \mid s\left(\alpha_{H}\right) \in S \alpha_{H}, H \in \mathscr{A}_{X}\right\}$. Then $D(\varnothing) / D(X)$ $\simeq F_{X} / F_{X}^{0}$.

Proof. Recall that $D(\varnothing)$ is isomorphic to the $S$-module of all linear maps $V^{*} \rightarrow S$ and $D(X)$ corresponds to the submodule of it of all maps which send 
$\alpha_{H}$ to $S \alpha_{H}$ for every $H \in \mathscr{A}_{X}$. Both these modules have as a direct summand the $S$-module of all linear maps $\overline{\operatorname{Ann} X} \rightarrow S$ where $\overline{\operatorname{Ann} X}$ is a fixed linear complement of Ann $X$ in $V^{*}$. Cancelling this summand we obtain the result.

Lemma 4.5. Let $X \in L$ be such that $\mathscr{A}_{X}$ is independent (i.e., Boolean). Then

$$
D(\varnothing) / D(X)=\bigoplus_{H \in \mathscr{A}_{X}} D(\varnothing) / D(H) .
$$

Proof. The result follows immediately from Lemma 4.4 and the equality

$$
\text { Ann } X=\bigoplus_{H \in \mathscr{A}_{X}} K \alpha_{H}=\bigoplus_{H \in \mathscr{A}_{X}} \text { Ann } H \text {. }
$$

Below we will identify the modules $D(\varnothing) / D(X)$ and $F_{X} / F_{X}^{0}$ via the isomorphism from Lemma 4.4.

Proposition 4.6. Consider the $S$-module $F$ of all linear maps $s: C_{1} \rightarrow S$ satisfying the two conditions (A) $s\left(E_{1}\right)=0$ and (B) $s\left(X_{1}, H\right)-s\left(X_{2}, H\right) \in S \alpha_{H}$ for every $X_{1}, X_{2} \in M$ and $H \supset X_{1} \cup X_{2}$. Put $F^{0}=\{s \in F \mid s(X, H) \in$ $\left.S \alpha_{H},(X, H) \in C_{1}\right\}$. Then $\Gamma \simeq F / F^{0}$.

Proof. Let $\gamma \in \Gamma$. For every $X \in M$ choose a representative $\widetilde{\gamma(X)} \in F_{X}$ of $\gamma(X)$. Then define the linear map $s=s_{y}: C_{1} \rightarrow S$ by $S(X, H)=\widetilde{\gamma(X)\left(\alpha_{H}\right)}$ $\left((X, H) \in C_{1}\right)$. Clearly $s$ annihilates $R_{X}$ and the property (A) of $s$ follows immediately. Now if $X_{1}$ and $X_{2}$ are in $M$ and $H \supset X_{1} \cup X_{2}$ then $\gamma\left(X_{1}\right)-$ $\gamma\left(X_{2}\right) \in D(H)$ which implies (B). Thus $s_{\gamma} \in F$.

If in the above construction we choose other representatives of $\gamma(X)$ in $F_{X}$ then $s_{\gamma}$ changes by an element of $F^{0}$. Thus the assignment $\gamma \mapsto s_{\gamma}+F^{0}$ defines a linear map $\Gamma \rightarrow F / F^{0}$. The injectivity of this map is immediate.

The rest of the proof is devoted to the surjectivity of this map. Let a map $s: C_{1} \rightarrow S$ belong to $F$, i.e., satisfy the conditions (A) and (B). We want to construct $\gamma \in \Gamma$ such that $s_{\gamma}=s$. Notice that $s_{\gamma}$ is defined already by the restriction of $\gamma$ to $L(2)$. On the other hand due to (4.1)' it suffices to construct $\gamma$ only on $L(2)$. For every $H \in \mathscr{A}$ we define the linear map $\widetilde{\gamma(H)}$ : Ann $H \rightarrow$ $S$ by $\widetilde{\gamma(H)}\left(\alpha_{H}\right)=s(X, H)$ for some $X \in M$ such that $X \subset H$. Due to (B) and Lemma 4.4 the class $\gamma(H)=\gamma(\widetilde{\gamma})+D(H)$ in $D(\varnothing) / D(H)$ does not depend on the choice of $X$. Now for every $X \in M$ we define the linear map $\gamma\left(\widetilde{\gamma(X)}\right.$ : Ann $X \rightarrow S$ by $\widetilde{\gamma(X)}\left(\alpha_{H}\right)=s(X, H), H \in \mathscr{A}_{X}$. This map is well defined because of (A) and we again put $\gamma(X)=\widetilde{\gamma(X)}+D(X)$. Finally if $X \in L(2) \backslash(M \cup \mathscr{A})$ then $\mathscr{A}_{X}$ is independent, i.e., consists of two elements, say $H_{1}$ and $H_{2}$. Then we define $\gamma(X)$ as $\left(\gamma\left(H_{1}\right), \gamma\left(H_{2}\right)\right)$ using Lemma 4.5. It is easy to see that the collection $\gamma=(\gamma(X))_{X \in L(2)}$ is an element from $\Gamma\left(L(2), \mathscr{D}^{\prime}\right)$ which can be identified with $\Gamma$ due to $(4.1)^{\prime}$. Clearly $s_{\gamma}=s$ which completes the proof. 4.6.

Below we will identify $\Gamma$ and $F / F^{0}$ via the isomorphism from Proposition

Proposition 4.7. Let $\gamma \in \Gamma$. Then $\gamma \in \operatorname{Im} \pi$ if and only if there exists a representative $s_{\gamma} \in F$ of $\gamma$ such that $s_{\gamma}=\bar{s} \rho$ for some $\bar{s}: E_{0} \rightarrow S$. 
Proof. Recall that $\operatorname{Im} \pi \simeq D(\varnothing) / D(U)$ and according to Lemma $4.4 D(\varnothing) / D(U)$ $=F_{U} / F_{U}^{0}$. Also notice that $F_{U}$ is the module of all linear maps $V^{*} \rightarrow S$ or equivalently all linear maps $E_{0} \rightarrow S$ annihilating $\operatorname{Im} \tau$. The result follows.

Now we continue with the setup for the main result. Despite many relations between the spaces of the complexes $C$ and $E$ there is no natural chain map between these complexes. To have such a map we consider still another complex $E \otimes V: 0 \rightarrow E_{1} \otimes V \stackrel{\tau \otimes 1}{\longrightarrow} E_{0} \otimes V \rightarrow 0$ and the maps $\nu_{0}: E_{0} \otimes V \rightarrow C_{0}$ and $\nu_{1}: C_{1} \otimes V \rightarrow C_{1}$ defined by $\nu_{0}(H \otimes v)=\alpha_{H}(v) H \quad(H \in \mathscr{A}, v \in V)$ and $\nu_{1}((X, H) \otimes v)=\alpha_{H}(v)(X, H) \quad\left(X \in M, H \in \mathscr{A}_{X}, v \in V\right)$. We keep the notation $\nu_{1}$ for the restriction of $\nu_{1}$ to $E_{1} \otimes V$ and consider the following diagram.

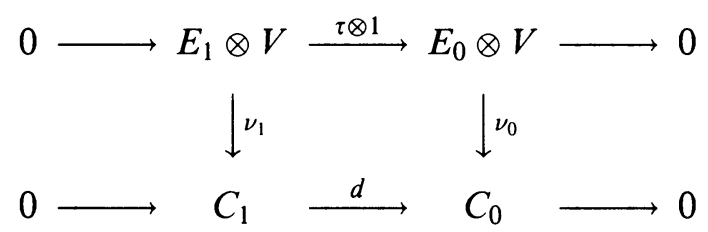

Lemma 4.8. The map $\nu=\left(\nu_{0}, \nu_{1}\right): E \otimes V \rightarrow C$ is a chain map, i.e., the above diagram is commutative.

Proof. Fix $X \in M, r=\sum_{H \in \mathscr{A}_{X}} c_{H} H \in R_{X}$, and $v \in V$. We have

$$
\nu_{0}(\tau \otimes 1)(r \otimes v)=\sum_{H \in \mathscr{A}_{X}} c_{H} \alpha_{H}(v) H
$$

and

$$
d \nu_{1}(r \otimes v)=\sum_{H \in \mathscr{A}_{X}} c_{H} \alpha_{H}(v) H-\left(\sum_{H \in \mathscr{A}_{X}} c_{H} \alpha_{H}(v)\right) X .
$$

The second sum in $(* *)$ is 0 because $r \in R_{X}$ and thus $\sum_{H \in \mathscr{A}_{X}} c_{H} \alpha_{H}=\lambda_{X}(r)=$ 0 . Comparing $(*)$ and $(* *)$ we obtain the result.

Notice that all $S$-module isomorphisms we have considered in this section are isomorphisms of graded modules. Now we focus our attention on the first degree components of these modules. The goal of the rest of the section is to prove the following result.

Theorem 4.9. Let $\mathscr{A}$ satisfy the conditions (4.1)-(4.3). Then the map $\pi: D(\varnothing)$ $\rightarrow \Gamma$ is surjective in degree 1 if and only if the map $\nu_{*}: H_{1}(E \otimes V)=T \otimes V \rightarrow$ $H_{1}(C)$ is surjective.

We denote the homogeneous component of degree 1 of the graded modules $\Gamma, F$, and $F^{0}$ by $\Gamma_{1}, F_{1}$, and $F_{1}^{0}$ respectively. The definitions of the spaces $F_{1}$ and $F_{1}^{0}$ coincide with the definitions of $F$ and $F^{0}$ from Proposition 4.6 except one has to substitute $S_{1}=V^{*}$ instead of $S$.

We also need to fix a (linear) section $\sigma: E_{0} \rightarrow C_{1}$ of $\rho$ which is possible since $\rho$ is surjective. For that we choose for every $H \in \mathscr{A}$ some $X_{H} \in M$ such that $H \supset X_{H}$ and put $\sigma(H)=\left(X_{H}, H\right)$. Notice that $\operatorname{Im} \sigma \cap H_{1}(C)=0$.

Lemma 4.10. Define the linear map $\phi: F_{1} \rightarrow C_{1}^{*}$ by $s \mapsto \delta_{s}$ where $s \in F_{1}$, $\delta_{s} \in C_{1}^{*}$, and $\delta_{s}(X, H) \alpha_{H}=s(X, H)-s\left(X_{H}, H\right)$ for every $(X, H) \in C_{1}$. Then $\operatorname{Im} \phi$ consists of all $\delta \in C_{1}^{*}$ such that (i) $\delta \sigma=0$ and (ii) $\delta\left(\nu_{1}(T \otimes V)\right)=0$. 
Proof. Let $s \in F_{1}$, i.e., $s: C_{1} \rightarrow V^{*}$ satisfying the conditions (A) and (B) of Proposition 4.6. Put $\delta=\delta_{s}$. The property (i) of $\delta$ is obvious. To prove the property (ii) fix $t=\sum c_{X}, H(X, H) \in T$ and $v \in V$. Notice that by definition of $T$ and $\tau$ we have $\sum_{X \subset H} c_{X, H}=0$ for every $H \in \mathscr{A}$. Thus

$$
\begin{aligned}
\delta \nu_{1}(t \otimes v) & =\sum c_{X, H} \alpha_{H}(v) \delta(X, H) \\
& =\left(s(t)-\sum c_{X, H} s\left(X_{H}, H\right)\right)(v) \\
& =\left(s(t)-\sum_{H}\left(\sum_{X \subset H} c_{X, H}\right) s\left(X_{H}, H\right)\right)(v)=0 .
\end{aligned}
$$

since $t \in T \subset E_{1}$ and $s$ satisfies (A).

Now suppose that $\delta \in C_{1}^{*}$ and satisfies (i) and (ii). Define a linear map $\Delta: C_{1} \rightarrow V^{*}$ by $\Delta(X, H)=\alpha_{H} \delta(X, H)$ for every $(X, H) \in C_{1}$. Notice that if $t \in T$ then for every $v \in V$ we have $\Delta(t)(v)=\delta \nu_{1}(t \otimes v)=0$ by (ii), i.e., $\Delta(T)=0$. Then define a linear map $s_{0}: E_{0} \rightarrow V^{*}$ satisfying the condition $s_{0}(\tau(r))=-\Delta(r)$ for every $r \in E_{1}$ and arbitrary otherwise. This map is well defined (although not uniquely) since $\Delta(T)=0$ and $T=\operatorname{ker} \tau$. Finally put $s(x)=\Delta(x)+s_{0}(\rho(x))$ for every $x \in C_{1}$. Clearly $s$ satisfies the conditions (A) and (B) and hence $s \in F_{1}$. Furthermore for every $(X, H) \in C_{1}$ we have

$$
\begin{aligned}
s(X, H)-s\left(X_{H}, H\right) & =\Delta(X, H)-\Delta\left(X_{H}, H\right) \\
& =\alpha_{H}\left(\delta(X, H)-\delta\left(X_{H}, H\right)\right) \\
& =\alpha_{H} \delta(X, H)
\end{aligned}
$$

since $\delta$ satisfies (i). Thus $\delta_{s}=\delta$ which completes the proof.

For the next step it is convenient to view $C_{1}^{*}$ as a part of the cochain complex $0 \rightarrow C_{0}^{*} \stackrel{\partial}{\longrightarrow} C_{1}^{*} \rightarrow 0$ where $\partial$ is dual to $d$. Then every $\delta \in C_{1}^{*}$ is a cocycle and defines a class $\tilde{\delta}$ of cohomology.

Lemma 4.11. Let $\gamma \in \Gamma_{1}$ and let $s$ be a representative of $\gamma$ in $F_{1}$. Then $\gamma \in \operatorname{Im} \pi$ if and only if $\widehat{\phi(s)}=0$.

Proof. Put $\delta=\phi(s)$. According to Proposition 4.7, $\gamma \in \operatorname{Im} \pi$ if and only if there exists a linear map $\bar{s}: E_{0} \rightarrow V^{*}$ such that $\bar{s} \rho \in F_{1}$ and $\bar{s} \rho-s \in F_{1}^{0}$. Clearly these properties of $\bar{s}$ are equivalent to the conditions: $(\overline{\mathrm{A}}) \bar{s} \tau=0$ and $(\overline{\mathrm{B}}) \bar{s}(H)-s(X, H) \in K \alpha_{H}$ for every $(X, H) \in C_{1}$. Define $\bar{\delta} \in C_{1}^{*}$ by $\bar{\delta}(X, H) \alpha_{H}=\bar{s}(H)-s(X, H)$.

The main property of $\bar{\delta}(X, H)$ is that it does not depend on $H$ (and thus can be denoted by $\bar{\delta}(X))$. Indeed the restrictions of $\bar{s}$ and $s$ to $E_{X}$ for some $X \in$ $M$ both annihilate $R_{X}$ due to $(\overline{\mathrm{A}})$ and $(\mathrm{A})$. Therefore the restriction of $\bar{s}-s$ to $E_{X}$ induces a linear map $\psi: \operatorname{Ann} X \rightarrow \operatorname{Ann} X$ which due to $(\overline{\mathrm{B}})$ preserves the one-dimensional subspaces generated by $\alpha_{H}, H \in \mathscr{A}_{X}$. By definition of $M$ the space Ann $X$ is 2-dimensional and there are at least 3 distinct subspaces generated by $\alpha_{H}$. Thus $\psi$ is the multiplication by a scalar which is equal to $\bar{\delta}(X)$.

Now define an element $\zeta \in C_{0}^{*}$ by $\zeta(X)=\bar{\delta}(X) \quad(X \in M)$ and $\zeta(H)=$ 
$\bar{\delta}\left(X_{H}\right) \quad(H \in \mathscr{A})$. Then for every $(X, H) \in C_{1}$ we have

$$
\begin{aligned}
\partial(\zeta)(X, H) \alpha_{H} & =(\zeta(H)-\zeta(X)) \alpha_{H}=\left(\bar{\delta}\left(X_{H}\right)-\bar{\delta}(X)\right) \alpha_{H} \\
& =s(X, H)-s\left(X_{H}, H\right)=\delta(X, H) \alpha_{H},
\end{aligned}
$$

i.e., $\partial(\zeta)=\delta$. Thus $\tilde{\delta}=0$.

Conversely, assume that $\tilde{\delta}=0$. Then there exists $\zeta \in C_{0}^{*}$ such that $\partial(\zeta)=$ $\delta$, i.e., $\zeta(H)-\zeta(X)=\delta(X, H)$ for every $(X, H) \in C_{1}$. Define a linear map $\bar{s}: E_{0} \rightarrow V^{*}$ by $\bar{s}(H)=\zeta(H) \alpha_{H}+s\left(X_{H}, H\right)$ for every $H \in \mathscr{A}$. Using the definition of $\phi$ and the condition on $\zeta$ we also can write $\bar{s}(H)=\zeta(X) \alpha_{H}+$ $s(X, H)$ for every $(X, H) \in C_{1}$. This implies $(\overline{\mathrm{B}})$ for $\bar{s}$. To check $(\overline{\mathrm{A}})$ for $\bar{s}$ fix $X \in M$ and $r=\sum c_{X, H}(X, H) \in R_{X}$. Then due to the condition (A) on $s$ and the definition of $R_{X}$ we have

$$
\begin{aligned}
\bar{s} \tau(r) & =\zeta(X) \sum_{H \in \mathscr{A}_{X}} c_{X, H} \alpha_{H}+s(r) \\
& =\zeta(X) \lambda_{X}(r)+s(r)=0
\end{aligned}
$$

which proves $(\overline{\mathrm{A}})$. Now the result follows from Proposition 4.7.

Proof of Theorem 4.9. Suppose $\nu_{1}(T \otimes V)=H_{1}(C)$. Consider an arbitrary $\gamma \in \Gamma_{1}$ and a representative $s \in F_{1}$ of $\gamma$. Since the functional $\delta=\phi(s) \in C_{1}^{*}$ satisfies the condition (ii) from Lemma 4.10 it annihilates $H_{1}(C)$. Thus $\tilde{\delta}=0$. Then due to Lemma $4.11 \gamma \in \operatorname{Im} \pi$.

Conversely suppose there exists $a \in H_{1}(C) \backslash \nu_{1}(T \otimes V)$. Since $\operatorname{Im} \sigma \cap H_{1}(C)=$ 0 there exists a linear functional $\delta$ on $C_{1}$ such that $\delta \sigma=0, \delta \nu_{1}(T \otimes V)=0$, and $\delta(a)=1$. Since $\delta$ satisfies the conditions (i) and (ii) of Lemma 4.10 there exists $s \in F_{1}$ such that $\phi(s)=\delta$. Put $\gamma=s+F_{1}^{0} \in \Gamma_{1}=F_{1} / F_{1}^{0}$. Since $\delta(a) \neq 0$ we have $\tilde{\delta} \neq 0$. Thus, due to Lemma 4.11, $\gamma \notin \operatorname{Im} \pi$ which concludes the proof.

Corollary 4.12. If $\mathscr{A}$ is free then $\nu_{1}(T \otimes V)=H_{1}(C)$. In particular $l \operatorname{dim} T \geq$ $\operatorname{dim} H_{1}(C)$.

Remark 4.13. (i) The following informal interpretation of the space $E_{1}$ and its subspace $T$ may be useful for applications of Theorem 4.9. While $E_{1}$ is generated by all linear dependences among $\alpha_{H}$ of length 3 , the space $T$ consists of all nontrivial linear dependences among these dependences. Here by trivial dependences we mean those which involve only the functionals $\alpha_{H}$ from Ann $X$ for some fixed $X \in M$.

(ii) In some cases Theorem 4.9 gives an immediate answer to the question whether $\pi: D(\varnothing) \rightarrow \Gamma\left(\mathscr{D}^{\prime}\right)$ is surjective in degree 1 (i.e., whether $H_{1}^{1}\left(L_{0}, \mathscr{D}\right)=$ $0)$.

If $T=0$ and $H_{1}(C) \neq 0$ then the answer is negative. For example, this is the case for the arrangement given by $x, y, z, x+y, x+y+z, x-z$ (char $K \neq 2$ ).

If $H_{1}(C)=0$ then the answer is positive. For example, this is the case for the arrangement given by $x, y, z, x+y, x-y, x+y+z$.

(iii) Clearly the complex $C$ is defined by the lattice $L$. It is also easy to see that for a formal arrangement $\operatorname{dim} T$ is defined by $L$.

However I make the following conjecture. 
Conjecture. The dimension of the space $\nu_{1}(T \otimes V)$ is not defined by $L$, i.e., is not a combinatorial characteristic of $\mathscr{A}$. Moreover the surjectivity of $\pi$ in degree 1 , i.e., the vanishing of the obstruction $H_{1}^{1}\left(L_{0}, \mathscr{D}\right)$ to the freeness is not a combinatorial property even for formal arrangements.

\section{ACKNOWLEDGMENT}

The author is grateful to M. Falk, H. Terao, and G. Ziegler for useful comments on his results and for sending him their unpublished work.

\section{BIBLIOGRAPHY}

[FR] M. Falk and R. Randell, On the homotopy theory of arrangements, Adv. Stud. Pure Math. 8 (1986), 101-124.

[G] R. Godement, Topologie algébrique et théorie des faisceaux, Hermann, Paris, 1958.

[ST] L. Solomon and H. Terao, A formula for the characteristic polynomial of an arrangement, Adv. in Math. 64 (1987), 305-325.

[T1] H. Terao, Arrangements of hyperplanes and their freeness. I, J. Fac. Sci. Univ. Tokyo Sect. IA Math. 27(2) (1980), 293-312.

[T2] _ Generalized exponents of a free arrangements of hyperplanes and Shephard-ToddBrieskorn formula, Invent. Math. 63 (1981), 159-179.

[T3] _ Free arrangements of hyperplanes over an arbitrary field, Proc. Japan Acad. 59 (1987), 301-304.

[Y] S. Yuzvinsky, Cohomology of local sheaves on arrangement lattices, Proc. Amer. Math. Soc. 112 (1991), 1207-1217.

[Z] G. Ziegler, Combinatorial construction of logarithmic differential forms, Adv. in Math. 76 (1989), 116-154.

Department of Mathematics, University of Oregon, Eugene, Oregon 97403

E-mail address: yuz@math.uoregon.edu 